\title{
The miniaturized pediatric continuous-flow device: Preclinical assessment in the chronic sheep model
}

\author{
Iki Adachi, MD, ${ }^{\text {a,b }}$ Sarah Burki, MD, a,b David Horne, MD, ${ }^{\text {a,b }}$ Gil G. Costas, DVM, ${ }^{\mathrm{c}}$ Taylor Spangler, DVM,
} Robert Jarvik, MD, ${ }^{\mathrm{e}}$ John Teal, BS, ${ }^{\mathrm{e}}$ J. Timothy Baldwin, $\mathrm{PhD},{ }^{\mathrm{f}}$ Kurt Dasse, $\mathrm{PhD},{ }^{\mathrm{g}}$ M. Patricia Massicotte, MD, ${ }^{\mathrm{h}}$ Jeff Conger, BS, ${ }^{\mathrm{c}}$ William E. Cohn, $\mathrm{MD},{ }^{\mathrm{c}}$ and Charles D. Fraser, Jr, $\mathrm{MD}^{\mathrm{a}, \mathrm{b}}$

\begin{abstract}
Background: The Infant Jarvik 2015 is an implantable axial-flow ventricular assist device (VAD) that has undergone the major evolutionary design modifications to improve hemocompatibility. This study was conducted in anticipation of data submission to the US Food and Drug Administration to obtain Investigational Device Exemption approval.

Methods: The VAD was implanted via a left thoracotomy in Barbado sheep $(\mathrm{n}=10,26(19-34] \mathrm{kg})$. Anticoagulation was maintained with coumadin, with a target international normalized ratio of greater than the individual sheep's baseline values. The VAD was managed at the highest possible speed as clinically tolerable. Complete necropsy was performed at the end of the study.

Results: There were 2 early mortalities: tension pneumothorax $(n=1)$ and shower emboli of the fragmented myocardium $(n=1)$. The remaining 8 sheep ( 2 with 30-day and 6 with 60-day protocols) completed the anticipated study duration in excellent condition, with the 6 completing 60-day sheep showing appropriate weight gain during support. There were no signs of clinically significant hemolysis, with the final plasma-free hemoglobin of 2 (1-17) mg/dL. Necropsy showed old renal infarction in 7 sheep. Although thromboembolism can be the potential etiology, given the mild anticoagulation regimen, other sources of emboli were identified in 2 sheep (graft coating material and fragmented myocardium). Flow study demonstrated favorable increase in flow (up to $3.0 \mathrm{~L} / \mathrm{min}$ ) in proportion to change in pump speed.
\end{abstract}

Conclusions: This study has demonstrated that the Infant Jarvik 2015 VAD is capable of maintaining its functionality for an extended period of time with minimal hemolysis. (J Thorac Cardiovasc Surg 2017;154:291-300)

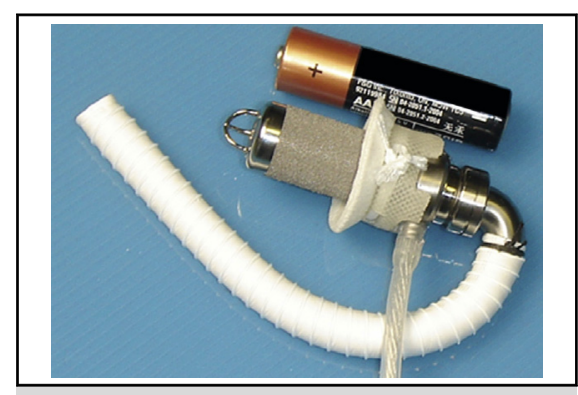

The Infant Jarvik 2015 ventricular assist device.

Central Message

This preclinical study has demonstrated the Infant Jarvik 2015 VAD maintains its functionality for an extended period of time with minimal hemolysis.

\section{Perspective}

Although continuous-flow devices are being used more often in children, pulsatile devices remain the only feasible options in infants due to size constraint. The Infant Jarvik 2015, the first continuous-flow device designed for small children, has demonstrated favorable hemocompatibility in this preclinical study. This study may herald the era of continuous-flow support in small children.

See Editorial Commentary page 301

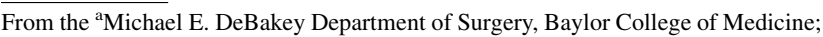
${ }^{\mathrm{b} C}$ Congenital Heart Surgery, Texas Children's Hospital; 'Texas Heart Institute, Houston, Tex; ${ }^{\mathrm{d}}$ VDx Preclinical, Davis, Calif; ${ }^{\mathrm{e} J a r v i k}$ Heart, Inc, New York, NY; ${ }_{\mathrm{f}}^{\mathrm{N}}$ ational Heart, Lung and Blood Institute, Bethesda, Md, ${ }^{\mathrm{g}} \mathrm{Geno}$, LLC, Cocoa, Fla; and hepartment of Pediatrics, University of Alberta, Edmonton, Alberta, Canada.

This study was funded by the National Heart, Lung, and Blood Institute's PumpKIN program.

Read at the 96th Annual Meeting of The American Association for Thoracic Surgery, May 14-18, 2016, Baltimore, Maryland.

Received for publication Aug 11, 2016; revisions received Nov 21, 2016; accepted for publication Dec 16, 2016; available ahead of print May 9, 2017.

Address for reprints: Iki Adachi, MD, Congenital Heart Surgery, Texas Children's Hospital, Baylor College of Medicine, 6621 Fannin St, WT19345H, Houston, TX 77030 (E-mail: iadachi@bcm.edu).

$0022-5223 / \$ 36.00$

Copyright (c) 2017 by The American Association for Thoracic Surgery

http://dx.doi.org/10.1016/j.jtcvs.2016.12.070
}

The infant Jarvik ventricular assist device (VAD) has been under development for more than a decade, as 1 of the 6 devices originally funded by the National Heart, Lung, and Blood Institute's Pediatric Circulatory Support program ${ }^{1}$ and subsequently by the PumpKIN program. ${ }^{2}$ The Infant Jarvik VAD is currently the only device remaining in the

Scanning this QR code will take you to a supplemental video. To view the AATS 2016 Webcast, see the URL next to the video thumbnail.

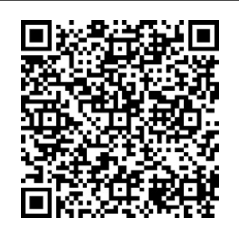




\author{
Abbreviations and Acronyms \\ $\mathrm{ACT}=$ activated clotting time \\ FDA $=$ Food and Drug Administration \\ GLP $=$ good laboratory practice \\ IDE = Investigational Device Exemption \\ INR $=$ international normalized ratio \\ $\mathrm{IV}=$ intravenous \\ $\mathrm{rpm}=$ revolutions per minute \\ $\mathrm{VAD}=$ ventricular assist device
}

program, which certainly highlights the extremely challenging nature of VAD development, especially those for pediatric use. In particular, development of an "implantable" VAD suitable for infants has been an especially demanding task. Although an infant VAD must meet all of the essential criteria required for adult VADs, the infant VAD also must function at a higher pump speed without increased blood damage despite much smaller blood flow channels and clearances than in the adult device. Infants VADs also require very fine motors, precise welds of paper-thin titanium components, and optimized hydrodynamic pump blade shapes for this unique heart failure population.

The initial approach was to scale down the adult Jarvik 2000; however, animal studies revealed that thrombosis occurred in the pin bearings used in the early prototypes that were as small as a AAA battery. The breakthrough to address this issue was the development of "cone" bearings that enabled the infant VAD to run long-term without bearing thrombosis. The product with the cone bearings was termed the "Infant Jarvik 2000 VAD" (11-mm outer diameter). The Infant Jarvik 2000 was extensively tested in an acute piglet model $(8 \mathrm{~kg})^{3}$ and in a chronic lamb model $(25 \mathrm{~kg})$ at a different institution. The data from these and other preclinical studies were submitted to the US Food and Drug Administration (FDA) to obtain Investigational Device Exemption (IDE) approval to initiate a clinical trial (the so-called PumpKIN trial) in August 2014. Unfortunately, in September 2014, the IDE approval was not granted, resulting in the postponement of the PumpKIN trial. The major concern raised by the FDA was significant hemolysis, as evidenced in the in vitro hemolysis test, coupled with the poor condition of the animals implanted with the Infant Jarvik 2000. Since then, the Infant Jarvik VAD has undergone major evolutionary design modifications, yielding a successful implantable infant-size VAD, named the Infant Jarvik 2015 VAD, which demonstrated a much improved hemolysis profile during in vitro testing. ${ }^{4}$ The specific aim of this study was to test biocompatibility and hemodynamic performance of the Infant Jarvik 2015 in a chronic sheep model, with a special emphasis on addressing concerns raised by the FDA in the previous preclinical studies.

\section{METHODS \\ Infant Jarvik VAD Design Change}

A pump optimization effort was initiated by identifying the actual source of hemolysis using computational fluid dynamics analysis, actual pump modifications, and bench hemolysis tests. Although hemolysis was determined to be substantially decreased at pump speeds below 20,000 rpm, the Infant Jarvik 2000 was unable to produce sufficient flow and pressure at the lower speeds. The decision was made to increase the pump outer diameter to $15 \mathrm{~mm}$ and to redesign the blades (Figure 1). With its larger flow channels, the new pump was designed to provide a flow of up to $3 \mathrm{~L} / \mathrm{min}$ at $18,000 \mathrm{rpm}$. The major differences of the old and new pumps are shown in Figure 2.

Hemocompatibility of the new pump was assessed by the bench hemolysis testing. There was more than 10-fold reduction in the normalized index of hemolysis down from $>0.6 \mathrm{~g} / 100 \mathrm{~L}$ with the old pump to $<0.04 \mathrm{~g} /$ $100 \mathrm{~L}$ with the new pump. The normalized index of hemolysis of the new pump is favorably compared with published data of the other devices previously developed. ${ }^{5,6}$ Based on these results, it was felt that the Infant Jarvik 2015 should be progressed to the next phase of testing: a preclinical trial in a chronic animal model.

\section{Animal Model}

The study was approved by the Texas Heart Institute's Institutional Animal Care and Use Committee, and all sheep received humane care in accordance with Principles of Laboratory Animal Care (National Society of Medical Research) and the Guide for the Care and Use of Laboratory Animals (National Institutes of Health). We planned to complete $30(+5)$ days support with the VAD in the first 2 sheep as a non-GLP (Good Laboratory Practice $),{ }^{7}$ and $60(+10)$ days support in the subsequent 8 sheep as a GLP study. In the two 30-day sheep, VAD flow measurement was conducted intraoperatively at the completion of the study immediately before euthanasia. Via a repeat left thoracotomy, the outflow graft of the VAD was exposed. A transonic flow probe (Transonic Systems, Inc, Ithaca, $\mathrm{NY}$ ) was placed on the graft. VAD flow was measured at different levels of pump speed, starting from 11,000 to $18,000 \mathrm{rpm}$.

\section{Implantation Procedure}

In each case, the sheep was placed in a stanchion for acclimatization before surgery. Food was withheld approximately 8 to 10 hours before surgery and water was available ad libitum. An intravenous (IV) line was placed in the right internal jugular vein for hydration. The sheep was premedicated with glycopyrrolate (0.01-0.025 mg/kg subcutaneous) and

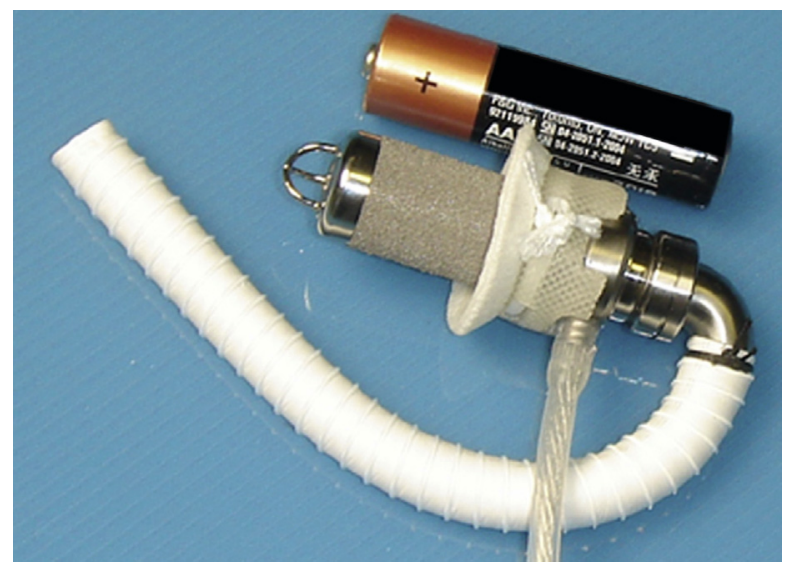

FIGURE 1. The Infant Jarvik 2015 ventricular assist device. 


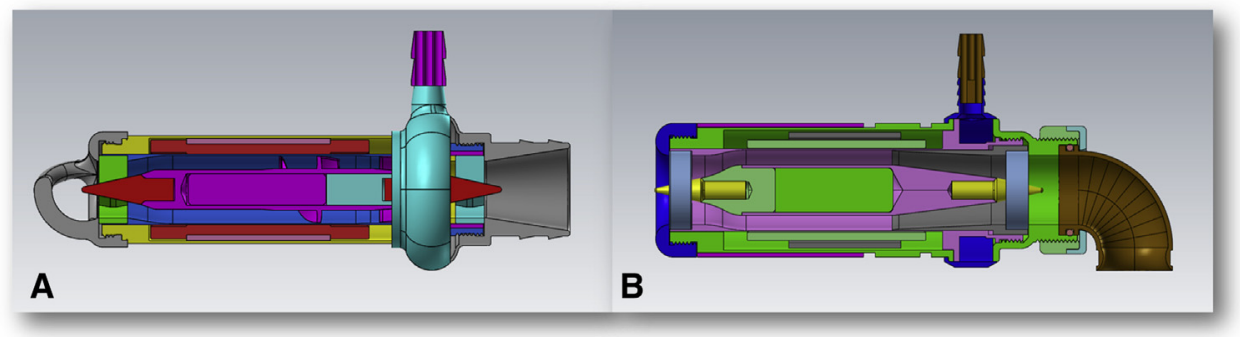

FIGURE 2. Design changes of Infant Jarvik VAD. A, Infant Jarvik 2000. Outer diameter $11 \mathrm{~mm}$, straight outflow with $8 \mathrm{~mm}$ ePTFE graft, flow rate between 0.5 and $1.5 \mathrm{~L} / \mathrm{min}$. B, Infant Jarvik 2015. Outer diameter $15 \mathrm{~mm}, 90^{\circ}$ curved outflow with $8 \mathrm{~mm}$ woven Dacron graft, flow rate between 0.5 and $3.0 \mathrm{~L} / \mathrm{min}$.

diazepam (0.1-2.0 mg/kg IV). Ketamine $(5-20 \mathrm{mg} / \mathrm{kg} \mathrm{IV})$ was used to induce sedation. The sheep was then intubated using a cuffed endotracheal tube, and the IV line replaced with a triple-lumen central venous catheter for administration of medication and monitoring of central venous pressure. The operative site was shaved and washed, following which the sheep was transported to the operating room. After administration of general anesthesia (isoflurane $0.5 \%-3.0 \%$, propofol $0.1-1.0 \mathrm{mg} / \mathrm{kg} / \mathrm{min} \mathrm{IV}$ ), an orogastric tube was placed to permit gastric decompression. The sheep was placed in a right lateral recumbency position, prepped, and draped in a sterile manner. An arterial line was inserted in the left common carotid artery with the cut-down technique. A left thoracotomy was performed via the sixth left intercostal space. The sixth rib was excised. The pulmonary inferior ligament was taken down for better mobility of the left lung. Proximal descending aorta just distal to the origin of the neck vessels was circumferentially dissected and encircled with an umbilical tape. The pericardium was opened longitudinally all the way down to its diaphragmatic reflection and superiorly to the level of the great arteries. A pericardial well was created by placing stay sutures along the pericardial edges. The VAD was brought into the field, and the driveline tunneled to the skin exit site close to the spine via the eighth intercostal space. The outflow graft was trimmed to an appropriate length. Heparin (initial dose: 100 units/kg and additional doses given based on activated clotting times [ACTs]) was administered. Having confirmed appropriate increase in ACTs $(>200)$, a partial occlusion clamp was placed on the proximal descending aorta. The outflow graft anastomosis was completed using a running 5-0 Prolene suture in an end-to-side fashion. The occlusion clamp was temporarily removed for de-airing through the VAD. By that time, calcium infusion and vasopressors (typically norepinephrine), as well as lidocaine infusion, had been started for hemodynamic support in preparation for inflow cannula insertion. Blood transfusion also was started in most cases during this period. The left ventricular apex was carefully lifted up by placing moist sponges underneath the cardiac mass. Some of the animals had very low tolerance for this maneuver, and became hemodynamically unstable. In such circumstances, an attempt was made to stabilize hemodynamics by optimizing pharmacological support and blood transfusion before proceeding with VAD implantation. Once satisfied with hemodynamics and exposure, the sewing ring was anchored to the left ventricular apex using 12 to 14 pairs of pledgetted 4-0 Prolene sutures. A cruciate incision was made in the left ventricular apex within the sewing ring by using a \#11 scalpel. The left ventricular apical core was removed using the proprietary coring knife. Following the coring of the left ventricle, the VAD was quickly inserted into the left ventricular cavity and secured to the sewing ring by tying the umbilical tapes, which were preattached to the sewing ring. Once the device was securely positioned in the ring, the cardiac apex was returned to its anatomical position. A 19-gauge needle was used to pierce the outflow graft proximal to the vascular clamp for de-airing. VAD support was initiated at the lowest-speed setting $(11,000 \mathrm{rpm})$. Once satisfied with de-airing, the vascular clamp was removed to establish VAD support. The VAD speed was uptitrated as appropriate by monitoring hemodynamics and power consumption indicator on the VAD controller. Chest tubes were placed in the pleural space. In the last 4 animals, the right pleural space was entered via the left thoracotomy and a 24-Fr Blake drain (Ethicon US LLC, Somerville, NJ) was placed in the right pleural cavity. After hemostasis was ensured, the adjoining ribs were approximated using sternal wires. Protamine was not used to counteract the effects of heparin. A subcutaneous catheter was placed for delivery of local anesthetics. The wound was then closed in a standard fashion.

Notably, from the surgical perspective, ventricular coring was the most challenging aspect of the operation: precipitation of ventricular fibrillation was not uncommon during this maneuver. Once fibrillated, successful conversion to normal rhythm (with electrocardioversion) was unlikely to occur. Therefore, optimization of hemodynamics before the coring procedure was greatly emphasized. If ventricular fibrillation developed and the standard electrocardioversion failed, direct cardiac massage was performed; simultaneously, the VAD was inserted and support commenced immediately to prevent irrecoverable left heart distension. This strategy allowed us to rescue 2 sheep that developed ventricular fibrillation.

\section{Postoperative Management}

Postoperatively, the sheep were housed in individual stanchions and monitored constantly in the laboratory's intensive care unit. Vital signs and observations were recorded hourly. Sheep were extubated after admission when appropriate. In typical cases in which the intraoperative course was smooth, sheep usually became tachycardic and hypertensive at this stage. Inotropes, if being infused, were aggressively weaned and, instead, a vasodilator and/or beta-blocker infusion was started during this period. However, in sheep with more complicated intraoperative course, such as the ones with ventricular fibrillation during left ventricular coring, inotropic support was continued longer according to the clinical necessity. Oral feeding was resumed 3 hours after extubation. Chest tubes were removed between postoperative days 2 to 4 , when the total drainage became less than $3 \mathrm{~mL} / \mathrm{kg}$ per day. Arterial pressures and central venous pressure were monitored for a median of 5 to 8 days, after which the arterial catheter and the central venous catheter were removed. Antibiotics were discontinued when these catheters were removed. The catheter used for paravertebral block was also usually removed at the end of the first postoperative week; subsequently, oral analgesics were given for pain control. Heart rate and rhythm were monitored via electrocardiogram for the entirety of the study. In most of the sheep, oral beta-blocker (bisoprolol) was given to maintain a resting heart rate of 100 to 120 per minute. Laboratory investigations were performed every day for the first week, and then weekly until the completion of study. Additional laboratory tests were conducted when clinically indicated. No imaging studies, such as radiographs and echocardiography, were available for this study. Urine output was measured hourly. Gastroprotective agents, omeprazole and probiotic gel, were administered daily. The sheep were weighed once every 2 weeks to monitor health status.

\section{Anticoagulation Management}

Anticoagulation management in this study evolved over time. A heparin bridge (initiated with 10 units $/ \mathrm{kg} / \mathrm{h}$ and uptitrated to 20 units $/ \mathrm{kg} / \mathrm{h}$ ) was 


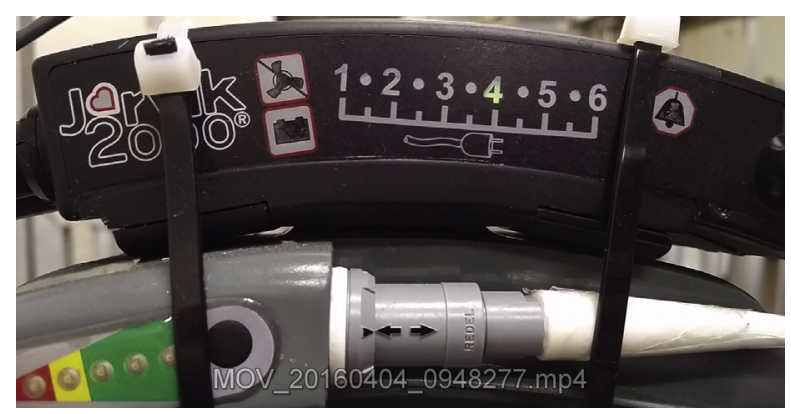

VIDEO 1. Fluctuation in pump power consumption. Video available at: http://www.jtcvsonline.org/article/S0022-5223(17)30412-9/addons.

used in the first 3 sheep. In the remaining sheep, coumadin was commenced directly without heparin bridge once the sheep started eating and defecating as normal. Antiplatelet agents were not given. Given the wide variation in the baseline international normalized ratio (INR) of the sheep (1.68-3.01), we elected to take a more individualized approach rather than to have a certain fixed goal. The goal was to maintain INR above the individual sheep's baseline value. To describe the efficacy of anticoagulation management, we used the Rosendaal method, ${ }^{8}$ which would demonstrate how much time each individual sheep had been in the range of target INR. This was assessed by calculating the percentage of days in which the sheep's INR was above its individual baseline INR level.

\section{VAD Management}

The Infant Jarvik 2015 VAD control console consists of a rotating dial that can be used to adjust the VAD speed, and an analog gauge showing wattage (ie, the power required by the impeller to maintain speed). The change in power consumption per this gauge (ie, pulsatility, the difference between the lowest and highest power levels; Video 1) indicates the periodic change in the blood volume flowing through the VAD during cardiac cycle. In other words, minimal VAD pulsatility with high pump speed would indicate a nearly sucked down left ventricular cavity. To avoid such situation, the goal of VAD management was to maintain the speed of the VAD as high as clinically tolerable, while maintaining VAD pulsatility of 1.0 Watt or greater.

\section{Necropsy}

At the end of each study, the sheep were heparinized and humanely euthanized in a manner consistent with the American Veterinary Medical Association Guidelines on Euthanasia, 2013. ${ }^{9}$ A complete fresh necropsy was performed by an experienced third-party veterinary pathologist (TS) who was blind to the specifics of each sheep's clinical course.

For necropsy, the sheep was placed in the right lateral recumbency position. As part of the external examination, the driveline exit site was inspected for signs of infection. The course of the outflow graft along the lateral surface of the left lung was observed for kinks. The heart (with device in situ), the outflow graft-descending aorta anastomosis, and a segment of the descending aorta were removed as a contiguous unit to allow gross and histological analysis of the VAD pathway for thrombosis. End organs, such adrenal glands, kidneys, liver, and spleen, were removed; the last step of the gross necropsy involved removing the calvarium, whereby the brain with cervical spinal cord were removed intact, all for similar analysis.

All explanted organs were then sectioned for intraparenchymal inspection. The heart was transected through the ventricles, just above the pump inflow, to allow assessment of pump-endocardium interface and intraventricular thrombosis. The pump was dismantled and outflow graft cut along its length to assess for presence of thrombi. Sections were taken from the aforementioned organs and fixed in 10\% neutral buffered formalin for histopathological analysis. Any tissue fragments found in the pump or the outflow pathway also were examined histologically.

\section{RESULTS \\ Overall Outcomes}

In total, 10 sheep were enrolled in the study (Table 1). The sheep were coded based on the study duration (30 or 60 days) and chronological order of surgery (eg, 60D-1 denotes the first sheep enrolled under the 60-day protocol, 60D-2 denotes the second sheep, and so on). Two sheep (both in the 60-day protocol) died early: sheep 60D-3 died of undiagnosed tension pneumothorax that occurred in the nonoperative, contralateral chest (right side) shortly after extubation. Necropsy study showed a large bulla at the right lung apex that had spontaneously ruptured presumably during mechanical ventilation. In sheep operated subsequent to this event, the right pleural space was entered via the left chest before the chest closure and an additional chest tube was placed in the right pleural space. The other sheep, 60D-5, had progressive abdominal distension postoperatively despite good hemodynamics and died on postoperative day 7. Necropsy study showed a complete obstruction of the small intestine, resulting from embolus of fragmented myocardium (detailed in a subsequent section).

The remaining 8 sheep ( 2 for the 30 -day and 6 for the 60 day protocol) completed the targeted study endpoints. Support duration ranged between 30 and 66 days (Table 1). There were no instances of gastrointestinal bleeding, renal

TABLE 1. Baseline characteristics of the study cohort

\begin{tabular}{lclcc}
\hline Animal ID & Baseline weight, kg & Outcome & Support duration, d & Final revolutions per minute \\
\hline 30D-1 & 34 & Completed & 31 & $18 \mathrm{~K}$ \\
30D-2 & 31 & Completed & 30 & $14 \mathrm{~K}$ \\
60D-1 & 29 & Completed & 65 & $18 \mathrm{~K}$ \\
60D-2 & 28 & Completed & 64 & $17 \mathrm{~K}$ \\
60D-3 & 20 & Died & 0 & N/A \\
60D-4 & 19 & Completed & 63 & N/A \\
60D-5 & 22 & Died & 7 & $16 \mathrm{~K}$ \\
60D-6 & 27 & Completed & 61 & $18 \mathrm{~K}$ \\
60D-7 & 26 & Completed & 66 & $16 \mathrm{~K}$ \\
60D-8 & 21 & Completed & & \\
\hline
\end{tabular}

N/A, Not applicable. 


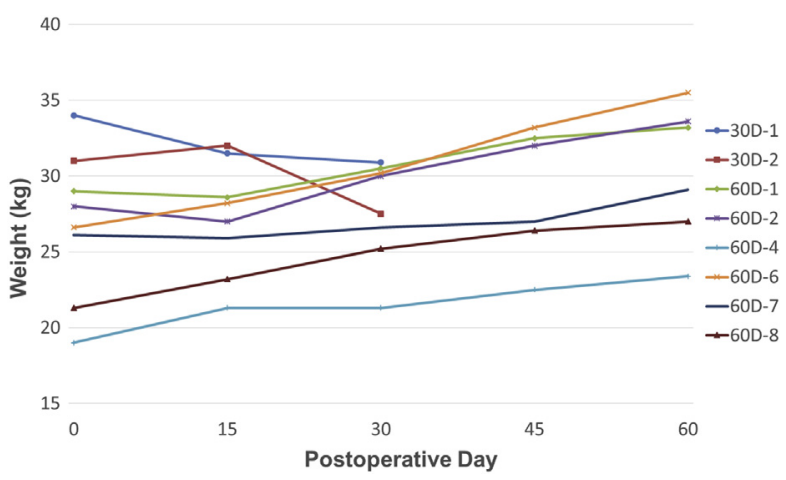

FIGURE 3. Serial changes in sheep weight during ventricular assist device support.

failure, or neurological dysfunction in this series. None of the sheep developed clinically significant infectious complications. These sheep had remained in a healthy state with good appetite and physical activity, as represented by favorable weight gain during VAD support in most of the sheep (Figure 3). Both of the two 30-day sheep had minor weight loss during VAD support despite good clinical status; this could have been attributed to substantial weight gain they had experienced before the VAD implantation. Due to the delay in initiation of the current study, these sheep gained weight substantially (30D-1: 27 to $34 \mathrm{~kg}$, 30D-2: 28 to $31 \mathrm{~kg}$ ) since their arrival to the laboratory 12 and 13 months prior, respectively.

\section{Hemocompatibility}

We serially measured plasma-free hemoglobin, lactate dehydrogenase, and hematocrit levels. The trends of these parameters in surviving animals, as well as reference ranges for sheep, are shown in Figure 4. Once past the acute phase, these markers were stable and within the clinically acceptable ranges in the vast majority of the cases. One of the outliers was the 30D-2 sheep that had minor elevation of plasma-free hemoglobin in the last week of the study. This was the sheep that had very limited VAD pulsatility, presumably due to right heart failure related to complicated intraoperative course. Attempts were made to increase pump speed to $18,000 \mathrm{rpm}$ despite limited VAD pulsatility. This resulted in nearly complete loss of pulsatility (sucked down left ventricle) and elevation of plasma-free hemoglobin. Optimization of pump speed to $14,000 \mathrm{rpm}$ resulted in restoration of adequate pulsatility and rapid clearance of plasma-free hemoglobin. This could have been attributed to a subsequent drop in hematocrit.

\section{Flow Study}

Flow study was conducted at the completion of the study in both 30-day sheep. As demonstrated in Figure 5, there was incremental increase in VAD flow as pump speed was increased. With lower afterload condition (the 30D-1 sheep
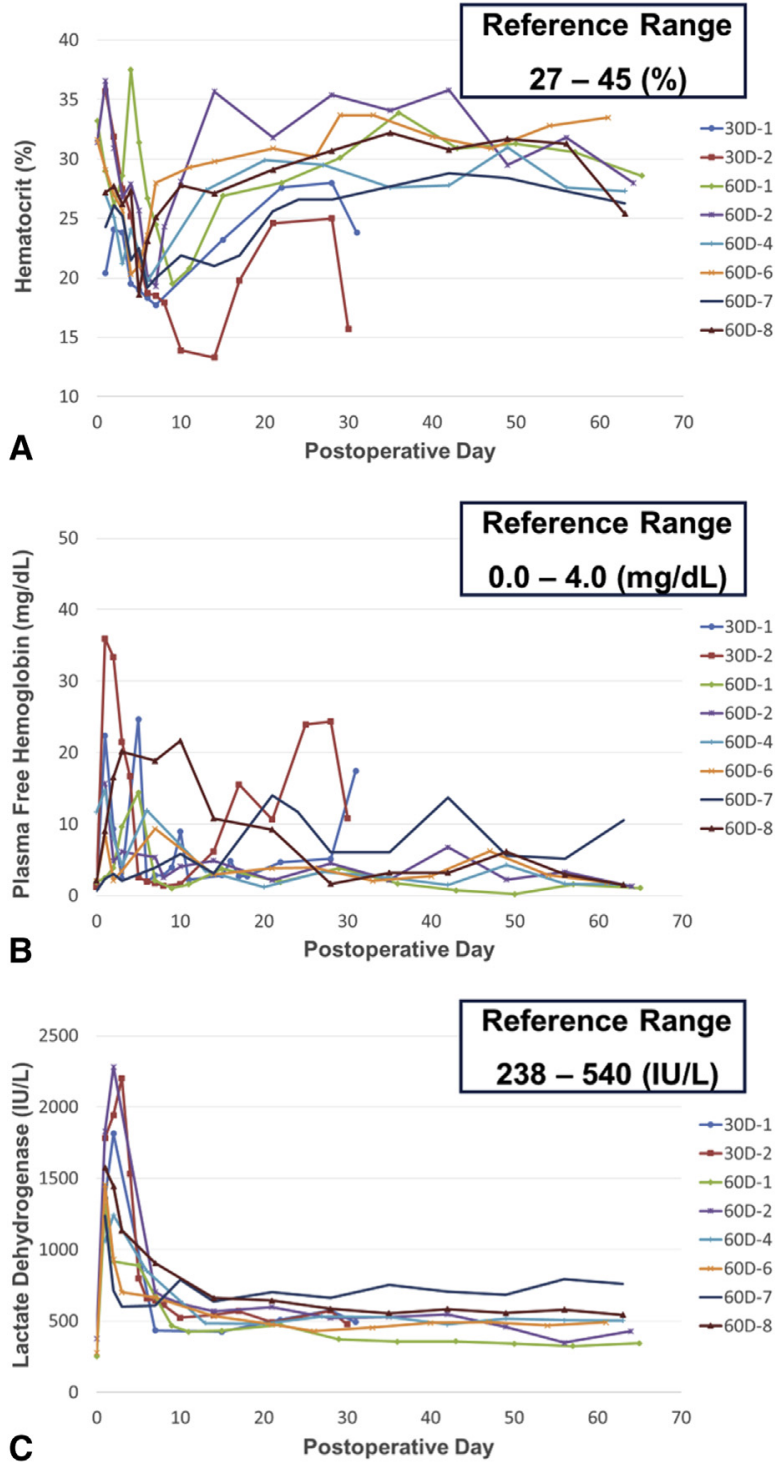

FIGURE 4. Hemocompatibility. Trends in hematocrit (A), plasma-free hemoglobin (B), and lactate dehydrogenase (C).

with an average mean arterial pressure of $76 \mathrm{~mm} \mathrm{Hg}$ ), the VAD flow reached $3 \mathrm{~L} / \mathrm{min}$ at the highest speed $(18,000 \mathrm{rpm})$. On the other hand, the VAD flow was maintained at $1 \mathrm{~L} / \mathrm{min}$ or less at the lower end of the VAD speed (11,000 to $12,000 \mathrm{rpm})$.

\section{Anticoagulation}

The ovine coagulation system remains poorly understood and described. During this study, it was noted that, in contrast to humans, ovine INRs demonstrated wide variability: baseline INR in our entire cohort varied between 1.68 and 3.01, and estimation of adequate anticoagulation over study duration was challenging. Overall, the sheep that completed the study spent an average of $48 \%$ of study 


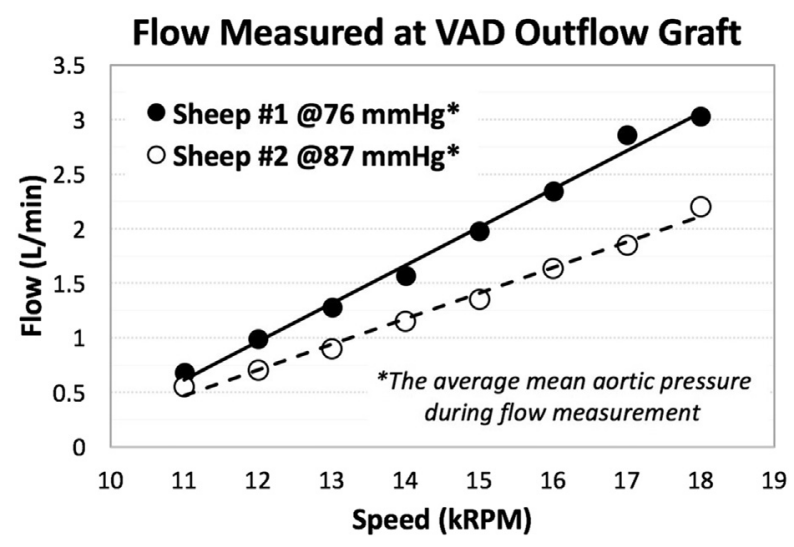

FIGURE 5. Flow capability. In vivo flow study was conducted at the conclusion of study in the 2 sheep with 30 -day protocol. There was a proportional increase in flow (up to $3 \mathrm{~L} / \mathrm{min}$ ) as ventricular assist device (VAD) speed was increased.

duration with INR above their individual baseline. In other words, the sheep's INR was below their individual baseline for $52 \%$ of the study duration.

\section{Necropsy}

In all sheep, the VAD was favorably placed in the center of left ventricular apex. A thin layer of smooth white fibrous pannus was observed to have developed on, and confined to, the sintered surface of the pump (Figure 6). The graft was positioned in a smooth, sloping curve along the lateral surface of the left lung, without any kinks, in all sheep.
Internally, the pump was clear of debris or thrombus in all but 3 sheep. In sheep 60D-4 and 60D-8, a small fibrin deposit ( 1 to $2 \mathrm{~mm}$ ) was found loosely attached to the pump stator. In sheep 60D-5, there was a fragmented piece of the myocardium identified in the pump housing. This was the sheep in which we had a significant difficulty when coring the left ventricle due to an extremely thick myocardial wall. Because an adequate opening was not achieved with the first attempt, the second coring was necessary in this case. The outflow graft was clear of debris or thrombus, and its anastomosis with the descending aorta was widely patent in all sheep.

Pale cortical lesions on the kidneys were commonly seen in this series. Three (30D-1, 60D-3, and 60D-6) had completely normal appearance of kidneys without such lesions, whereas the rest of the 7 sheep had at least 1 , but typically a couple of lesions with histological evidence of chronic ischemic infarction (Figure 5). In 2 cases (of the 7 with renal infarction), the actual emboli in the renal artery were identified. In 1 sheep (30D-2), focal embolization of gelatinous polymer materials was found in a right renal arteriole. This was believed to originate from a coating material of the outflow graft. This sheep also had evidence of chronic ischemic changes of the thalamus, right cerebellum, and the spinal cord. In the other sheep (60D-5) that had a fragmented piece of the myocardium in the pump housing as mentioned previously, there was widespread embolic infarction in multiple organs,

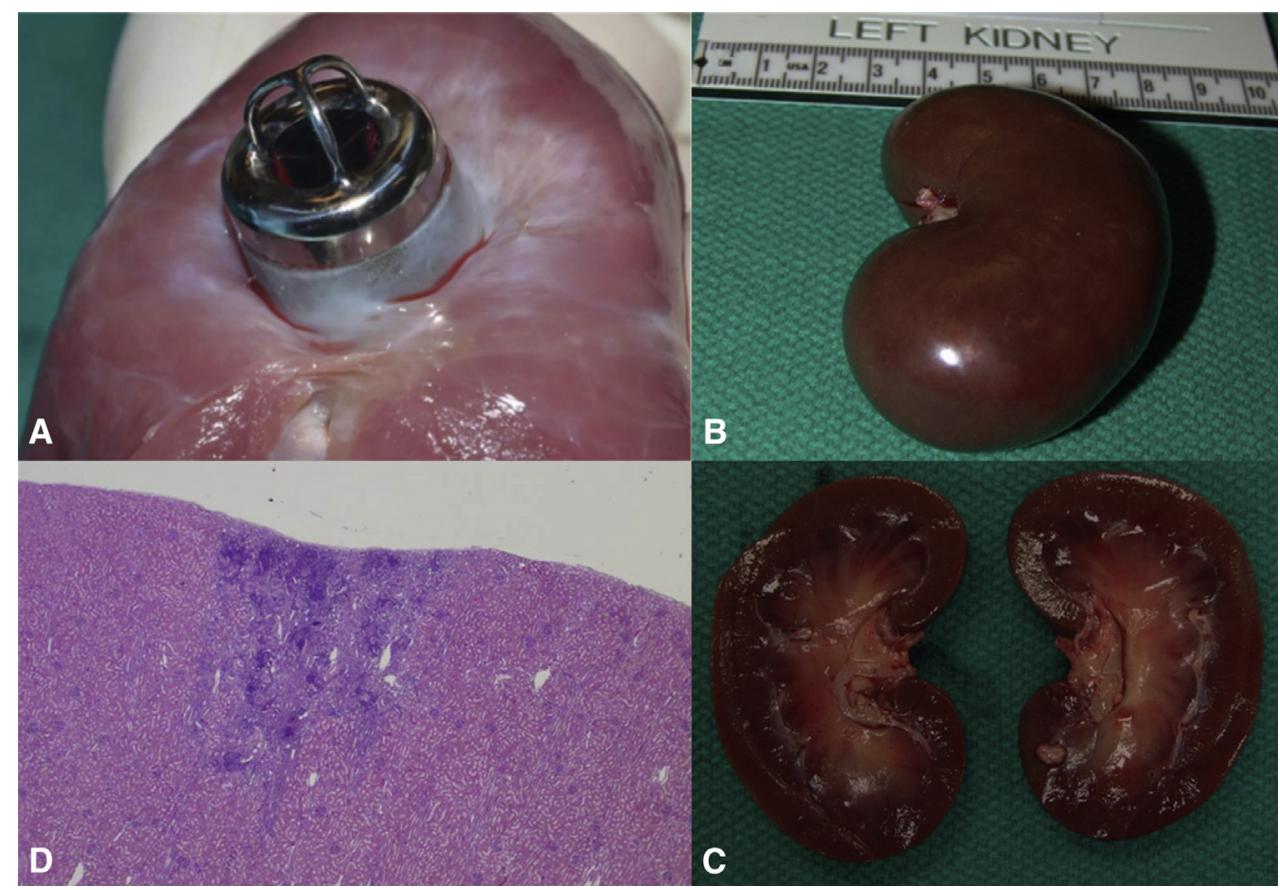

FIGURE 6. Necropsy. Representative necropsy findings. A, Smooth white pannus on the sintered external surface of the Infant Jarvik 2015 VAD without encroachment on the inflow ostium. B and C, Appearance of renal infarcts on gross necropsy. D, Photomicrograph of histological examination of renal infarct noted on gross necropsy (hematoxylin and eosin stain, original magnification $\times 2$ ). Small focus of tubular atrophy and inflammation consistent with a small chronic, resolved infarct. 
including kidney, liver, spleen, and small intestine, but not in the brain. Infarction of the small intestine and resultant intestinal obstruction was the direct cause of death in this sheep. In the rest of the 5 sheep with old renal infarction, the actual causes of kidney lesions were not identifiable.

\section{DISCUSSION}

There has been an increasing interest in the use of continuous-flow VADs in the pediatric population. Currently, half of the long-term devices reported to the PediMACS, which is the pediatric portion of the North American registry for VAD support, are continuous-flow VADs. ${ }^{10}$ The emergence of the HeartWare HVAD (Medtronic Inc, Fridley, MN), which is significantly smaller than previous devices, has accelerated this trend. ${ }^{11,12}$ The current wave of continuous-flow VAD support, nonetheless, has not yet reached small children due to size constraints and limited market of such a specialized device. Development of a small continuous-flow VAD, such as the Infant Jarvik 2015, has the potential to induce paradigm shift to the field of pediatric mechanical circulatory support that currently has limited device options.

In this study, we have demonstrated that the Infant Jarvik 2015 VAD is capable of maintaining its functionality for an extended period of time with minimal hemolysis. This is despite that the VAD was kept at high rpm in the setting of mild anticoagulation regimen. The only event of mild hemolysis was related to excessively high pump speed $(18,000 \mathrm{rpm})$ in the setting of limited right heart cardiac output, which caused sucked down left ventricle, as evidenced by nearly "zero" pulsatility of VAD power consumption. This minor hemolysis resolved quickly once such VAD speed was optimized. It would be worth mentioning that even with this extreme pump management, the highest plasma hemoglobin in this sheep (except the immediate postoperative phase) was less than $30 \mathrm{mg} / \mathrm{dL}$, the cutoff value generally accepted as a marker for hemolysis. Most of the sheep (8 of 10) completed the expected study duration in a good health state, as suggested by the absence of weight loss, and indeed, weight gain. Such weight gain is somewhat greater than that which would have been experienced by unoperated sheep. Although the reason for this has not been investigated in a head-to-head comparison, we speculate that the weight gain experienced in this cohort is because of prolonged restraint in a stanchion. Sheep generally have a nervous disposition and are known to flail vigorously if a threat is perceived. To prevent injury or catastrophic device dislodgement, we restrained the sheep in a stanchion where the only movement afforded to them was in the up-down direction. Unoperated sheep, on the other hand, may roam in larger pens. Flow measurement study conducted in 2 sheep has shown the wide range of flow $(0.5$ to $3.0 \mathrm{~L} / \mathrm{min})$ the Infant Jarvik 2015 can provide. This flow range would cover pediatric patients that currently do not have access to an implantable VAD support (approximately with a weight of $15 \mathrm{~kg}$ or less). We believe that this study not only has sufficiently addressed the concerns the FDA had raised at the previous IDE application for the old Infant Jarvik 2000, but also has provided additional information relevant to future clinical use.

The most challenging part of this experiment stems from the fact that there are no perfect animal models that mimic pediatric heart failure. Although the VAD is intended for use in dilated hearts with severely depressed function, experiment animals do not have such diseased hearts. Although testing a VAD in a heart with normal dimension and function is not ideal, it is impractical to create a chronic heart failure animal model for VAD experiments. As far as we know, all the previous animal studies on VAD development did not induce heart failure before VAD implantation. ${ }^{3,13-15}$ The fact that the VAD is implanted in hearts with normal dimension also has an important implication to the selection of animal size to choose for the study. We used the Barbado sheep, with a weight of approximately 20 to $30 \mathrm{~kg}$. Unlike piglets or calves with this weight range, the Barbado sheep are amenable to chronic study without "outgrowing" the VAD. ${ }^{7}$ Although this weight range is seemingly larger than the potential human target, we did not feel it was feasible to use smaller animals due to the size constraints of the left ventricle. In fact, the left ventricular cavity in our sheep model was barely large enough to accommodate the VAD.

The lack of standard anticoagulation regimen for sheep was an additional challenge in conducting the study. In the absence of standard anticoagulation regimen, 2 approaches would have been possible: to anticoagulate aggressively in an effort to maximally protect the VAD from hemolysis and thrombosis, or to err on the side of mild anticoagulation to demonstrate acceptable biocompatibility even without such excessive protection. In the real clinical setting, the need for weaning, or even temporarily discontinuing, anticoagulation during long-term VAD support is not a rare event. We thought the latter approach with mild anticoagulation would provide more relevant information in view of future clinical use.

Renal infarction occurs frequently during long-term animal studies testing VADs. ${ }^{14,15}$ In the absence of histologically confirmed renal intravascular thrombus, determining the etiology of such infarcts remains speculative. In this study, 7 of the 10 sheep had visible pale lesions in the kidneys consistent with old infarcts. These lesions had widely varying appearance, ranging in size from $1 \mathrm{~mm}$ surface discolorations, to $2 \mathrm{~cm}$ depressed lesions. There were no detectable functional sequelae in any of these 7 cases; all sheep had normal renal function throughout the study period. Although the exact etiology of such renal lesions remains unclear, necropsy revealed interesting findings in some of the sheep with renal 
infarction. In sheep 30D-2, an embolus of homogenous gelatinous matrix, similar to the coating material of the inner surface of the outflow graft, was found in a right renal arteriole. Although whether or not this embolus was truly causative of the right renal infarct remains unclear, this finding may indicate that not all renal infarctions were necessarily due to thromboembolism. The presence of chronic ischemic changes in the thalamus, right cerebellum, and the spinal cord further endorses this notion. The location of the outflow graft anastomosis (descending aorta distal to the neck vessels) and a presumed antegrade cardiac output from the native heart make it unlikely for the VAD to cause embolic infarction to the brain. In the other sheep (60D-5), it was found that a fragmented piece of the myocardium caused widespread shower emboli to multiple organs, including kidney, liver, spleen, and small intestine. Given such a mild anticoagulation regimen, nevertheless, it would not be surprising if some of these lesions were due to thromboembolic events related to the VAD. The presence of a small fibrin deposit on the pump stator in 2 sheep may support such speculation. Certainly, sophisticated anticoagulation management, which we could not provide in this animal study, would be of critical importance when conducting a clinical trial.

As discussed earlier, the major limitation of the current study resides in the fact that the VAD was tested in the animal with a nondilated heart with normal function. As clinically known, ${ }^{16}$ however, VAD support is much more challenging if the heart has a small ventricular cavity and systolic function is preserved. In this regard, it is plausible to state that the VAD was tested in a more challenging condition in this study than would be used clinically. This is particularly true because the VAD was maintained at the highest possible speed in the setting of mild anticoagulation, both of which would not be ideal in terms of hemolysis prevention. With the encouraging improvement we have observed with this new device, we hope the clinical trial will begin in the near future. This study will be the important milestone for the new era of continuous-flow VAD support in infants and small children.

\section{Webcast}

You can watch a Webcast of this AATS meeting presentation by going to: http://webcast.aats.org/2016/Video/ Monday/05-16-16_Ballroom_III_1635_Adachi-800.mp4.

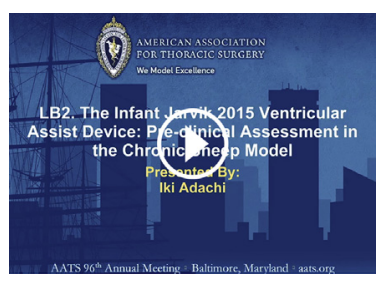

\section{Conflict of Interest Statement}

Dr Adachi serves as a consultant for the New England Research Institutes (Watertown, Mass) for this project. His employer, Texas Children's Hospital, receives salary support from the New England Research Institute for compensation. Dr Costas and Mr Conger are both employed by Texas Heart Institute, which was a subcontractor to Jarvik Heart, Inc. Dr Spangler received payment for provision of pathology services. Dr Jarvik is a significant shareholder of Jarvik Heart, Inc. Mr Teal is employed by Jarvik Heart, Inc, which received grants from the National Institutes of Health (NIH)/National Heart, Lung, and Blood Institute (NHLBI) for developing the Infant Jarvik 2015. Dr Massicotte is a consultant to the New England Research Institutes for the NHLBI PumpKIN Program. Dr Baldwin is an employee of the NHLBI. Dr Dasse is a member of the Executive Committee overseeing the NIH PumpKIN program. Drs Burki, Horne, and Fraser are employed by Texas Children's Hospital, which receives salary support from the New England Research Institute for Dr Adachi's role as study principal investigator. Dr Cohn has no conflicts of interest to declare. All other authors have nothing to disclose with regard to commercial support.

The authors acknowledge the efforts of Ms Kimberly Moody (research coordinator), Ms Cindy Van Riper (veterinary technician III), Mr Aaron Palmer (surgical technician), and the rest of the team at Texas Heart Institute in assisting with the conduct of this study. The results of this study were presented as an oral presentation at the 96th Annual Meeting of The American Association for Thoracic Surgery; May 14-18, 2016; Baltimore, Md.

\section{References}

1. Baldwin JT, Borovetz HS, Duncan BW, Gartner MJ, Jarvik RK, Weiss WJ, et al. The National Heart, Lung, and Blood Institute Pediatric Circulatory Support Program. Circulation. 2006;113:147-55

2. Baldwin JT, Borovetz HS, Duncan BW, Gartner MJ, Jarvik RK, Weiss WJ. The National Heart, Lung, and Blood Institute Pediatric Circulatory Support Program: a summary of the 5-year experience. Circulation. 2011;123: 1233-40.

3. Wei X, Li T, Li S, Sung Son H, Sanchez P, Niu S, et al. Pre-clinical evaluation of the infant Jarvik 2000 heart in a neonate piglet model. J Heart Lung Transplant. 2013;32:112-9.

4. Baldwin JT, Adachi I, Teal J, Almond CA, Jaquiss RD, Massicotte MP, et al. Closing in on the PumpKIN trial of the Jarvik 2015 ventricular assist device. Semin Thorac Cardiovasc Surg Pediatr Card Surg Annu. 2017;20: 9-15.

5. Sobieski MA, Giridharan GA, Ising M, Koenig SC, Slaughter MS. Blood trauma testing of CentriMag and RotaFlow centrifugal flow devices: a pilot study. Artif Organs. 2012;36:677-82.

6. Maul TM, Kocyildirim E, Johnson CA Jr, Daly AR, Olia SE, Woolley JR, et al. In vitro and in vivo performance evaluation of the second developmental version of the pediaflow pediatric ventricular assist device. Cardiovasc Eng Technol. 2011;2:253-62.

7. Food and Drug Administration. Good Laboratory Practice For Nonclinical Laboratory Studies. 2016. Available at: https://www.ecfr.gov/cgi-bin/text-idx? $\mathrm{SID}=73260131 \mathrm{c} 27 \mathrm{~b} 0 \mathrm{a} 88 \mathrm{ebfbbc} 4 \mathrm{c} 0995 \mathrm{dc} 06 \& \mathrm{mc}=$ true $\&$ node $=\mathrm{pt} 21.1 .58 \& \mathrm{rgn}=$ div5. Accessed March 31, 2017.

8. Rosendaal FR, Cannegieter SC, van der Meer FJ, Briet E. A method to determine the optimal intensity of oral anticoagulant therapy. Thromb Haemost. 1993;69: 236-9. 
9. Leary S, Underwood W, Anthony R, Cartner S, Corey D, Grandin T, et al, for the Panel on Euthanasia. AVMA Guidelines for the Euthanasia of Animals: 2013 Edition. Available at: https://www.avma.org/KB/Policies/Documents/ euthanasia.pdf. Accessed March 31, 2017.

10. Rossano JW, Lorts A, VanderPluym CJ, Jeewa A, Guleserian KJ, Bleiweis MS, et al. Outcomes of pediatric patients supported with continuous-flow ventricular assist devices: a report from the Pediatric Interagency Registry for Mechanical Circulatory Support (PediMACS). J Heart Lung Transplant. 2016;35:585-90

11. Kirklin JK, Naftel DC, Pagani FD, Kormos RL, Stevenson LW, Blume ED, et al. Sixth INTERMACS annual report: a 10,000-patient database. J Heart Lung Transplant. 2014;33:555-64.

12. Kirklin JK, Naftel DC, Pagani FD, Kormos RL, Stevenson LW, Blume ED, et al. Seventh INTERMACS annual report: 15,000 patients and counting. J Heart Lung Transplant. 2015;34:1495-504.

13. Gibber M, Wu ZJ, Chang WB, Bianchi G, Hu J, Garcia J, et al. In vivo experience of the child-size pediatric Jarvik 2000 heart: update. ASAIO J. 2010;56(4): 369-76.

14. Weiss WJ, Carney EL, Clark JB, Peterson R, Cooper TK, Nifong TP, et al. Chronic in vivo testing of the Penn State infant ventricular assist device. ASAIO J. 2012;58:65-72.

15. Cheung A, Chorpenning K, Tamez D, Shambaugh C Jr, Dierlam AE, Taskin ME, et al. Design concepts and preclinical results of a miniaturized HeartWare platform: the MVAD system. Innovations (Phila). 2015;10: 151-6.

16. Kirklin JK, Naftel DC, Kormos RL, Pagani FD, Myers SL, Stevenson LW, et al. Interagency Registry for Mechanically Assisted Circulatory Support (INTERMACS) analysis of pump thrombosis in the HeartMate II left ventricular assist device. J Heart Lung Transplant. 2014;33:12-22.

Key Words: ventricular assist device (VAD), continuousflow device, pediatrics, mechanical circulatory support (MCS), pediatrics heart transplantation

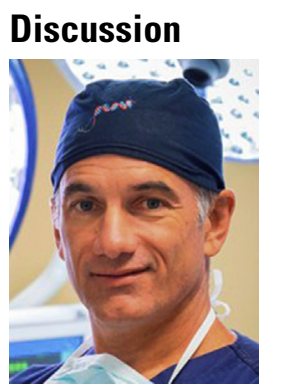

Dr C. Caldarone (Toronto, Ontario, Canada). Thank you, Dr Adachi, for bringing this important late-breaking clinical information to our attention.

For those of us who were at the symposium yesterday, this device was discussed, and I'll reiterate for those who weren't here, the device had an IDE application that was rejected in 2014 for hemolysis and since has undergone significant redesign and retesting, and a follow-up IDE application will be submitted. And if accepted, then a prospective trial comparing it to the Berlin Heart is anticipated, and this will have an important impact on our field. So congratulations to you for driving this forward and bringing it to our attention.

I do have a few questions, and this may betray my ignorance with regard to device testing and preparation for a clinical trial. But is a $30-\mathrm{kg}$ sheep appropriate for testing a device that's designed to be placed in an infant?

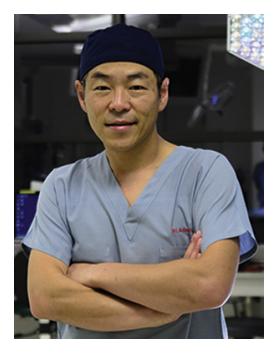

Dr Adachi (Houston, Tex). That's a very important question, and thank you very much, Dr Caldarone.

Actually, that's what I asked initially to the PumpKIN group when I was asked to do this study. The reality is that these animals have a very small LV cavity, and I don't really believe we can use smaller animals because of the limitation of the LV cavity. These animals have very hypertrophic left ventricular wall, and the cavity is so small.

So even though the animals are larger than the actual patients who would be supported with the infant Jarvik VAD, the animal's LV cavity would not be so much larger than the actual patient' heart, particularly because the patients would have dilated heart.

So just looking at the size of the heart, in particular the LV cavity, I think this animal model would be appropriate.

Dr Caldarone. And could you comment on using the device in a normal-ejecting heart as compared to a heart that might be dysfunctional?

Dr Adachi. I think that's also a very important question. Thank you.

The reality is that we can't really create a chronic heart failure model that completely resembles pediatric heart failure. There is no good way to develop the heart failure model. Even if we can develop such a model, it would be very difficult for the animal with significant heart failure to survive the operation we conducted in this study.

The operation is very traumatic, and out of 10 animals, 3 animals developed ventricular fibrillation while we were doing the ventricle coring. Just putting the knife in the left ventricular cavity caused ventricular fibrillation, and there was a lot of resuscitation efforts therefore.

These animals that had the problem during the operation limped a lot afterward. Fortunately, we were able to manage them and keep them alive. And subsequently they got better, and they gained weight, and they regained the healthy status.

The point I am trying to make is that if the animal already had significant heart failure, I don't believe the animals could survive the study.

The problem is we can't really do this type of operation through the sternotomy on pump because once we open the sternum, then they don't do well afterward. Unlike humans, the animals lie on the sternum while resting, and that's why the sternotomy is not a feasible option for chronic animal study. That's the major limitation of the study. Additionally, it is clinically known that VAD support in a nondilated heart with normal systolic function is more challenging than in a dysfunctional and dilated heart. In this regard, I may argue that the testing condition in this study would be considered to be more challenging for the device than the real clinical situation. 
Dr Caldarone. What are your thoughts about running the pump at flow rates that you might actually be using in an infant? I had your slides in advance but of course no manuscript, so it wasn't clear to me how flow was actually measured. And were the flow rates during the study conducted at a rate that would be commensurate with what might be flowed in an infant?

Dr Adachi. Thank you very much. That's also a very good question. We didn't put the flow probe during the device support in this animal model, and obviously this device doesn't have any flow estimation. So we didn't really know how much actually the device was flowing during the device support. That's why we performed the flow study in the 30-day animals before the termination of the study.

Based on the data we achieved from the flow study, most likely we were flowing between 1.5 to 2.5 liters in these animals during the study. Maybe some animal with the highest rpm, 18,000, we might have been flowing like close to 3 liters. That's all I can tell about the flow.

Regarding your question about the actual flow we would use for the clinical patient, most likely the flow our patient would need would be a little bit less than these animals had. And we don't know at this point whether we can run the pump safely at a relatively low rpm without having pump thrombosis.

That's why we are now planning to do a low-flow study in which we will run the pump at the lowest minimal rpm without having much retrograde flow through the device.

And, actually, in that study, we are planning to keep the flow probe in the chest for the entirety of the study so that we can monitor the flow continuously. I hope that study will provide a more accurate answer to your important question.

Dr Caldarone. I think that low-flow study and, of course, your final necropsy report to examine for how many embolic events you may have had in 420 days of testing, both those 2 pieces of data will probably be very important. Congratulations on some great work.

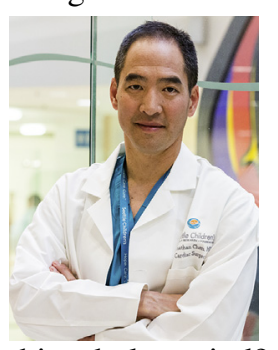

this whole period?
Dr J. Chen (Seattle, Wash). Iki, another great paper from your group and obviously moving the needle forward for all of us.

I just had some quick technical questions. Were any of your flows enough to cause the aortic valve to close, or was the animal completely ejecting during
Dr Adachi. Thank you, Dr Chen, for the important question. We can't really tell if the aortic valve was closed or not at what level rpm, because once we removed the arterial line, we obviously couldn't see the ejection. Also, we couldn't do echo. We tried, but we couldn't see anything.

Supposedly, these animals had normal ventricular function. Although most of the animals in this study were on beta blockers, cardiac function would still have been close to normal. Therefore, I think most of the animals were ejecting during the course of the support.

Dr Chen. Would you envision that the thoracotomy approach is the one that would be the clinical one, or do you think this would be a median sternotomy approach? As you know, the adults have sort of evolved back into a mini approach through a thoracotomy and for their continuous flow devices, and, of course, the original Jarviks were that way too.

So do think this would be standard, median sternotomy in the clinical trial for babies?

Dr Adachi. My personal opinion is that for clinical patients, I think the sternotomy approach with cardiopulmonary bypass is necessary so that we can see inside of the heart very well. That way, we can inspect the left ventricular cavity and remove trabeculations, if necessary, which might cause an obstruction to the device inflow during the course of the support.

The thoracotomy approach without bypass support is a very traumatic operation and physiologically a very demanding procedure for somebody with severe heart failure. I don't really believe the small infant with significant severe heart failure can tolerate that kind of operation.

Dr Chen. And the final question is, can you speculate a little bit on the lack of thrombus in any of the findings considering that the INR was essentially normal for most of this? Is it that the animal itself is just normally auto anticoagulated, and maybe this is the wrong model to be assessing that? Or as Chris was pointing out, is it at lower flows, if you continue to maintain essentially a normal INR, we'll start to see more clot?

Dr Adachi. That's a very difficult question to answer. I don't think I have a good answer for that because there is no standard in terms of how to anticoagulate these animals. Even though the INR is just above the baseline, maybe the animal had substantial anticoagulation effect. Sorry, I don't have the answer for that.

Dr Chen. Okay. Thank you. Great talk. 